\title{
Neutrinoless double beta decay search with KamLAND-Zen
}

\author{
Yoshihito Gando*† \\ Research Center for Neutrino Science, Tohoku University, Sendai 980-8578, Japan \\ E-mail: gando@awa.tohoku.ac.jp
}

\begin{abstract}
KamLAND-Zen is a neutrinoless double beta decay search experiment based on the ${ }^{136} \mathrm{Xe}$ loaded liquid scintillator. KamLAND-Zen 400 project was already terminated and its latest limit for half life of $0 v \beta \beta$ is $\mathrm{T}_{1 / 2}>1.07 \times 10^{26}$ years at $90 \%$ confidence level. It corresponds to Majorana effective mass $\left\langle m_{\beta \beta}\right\rangle<61-165 \mathrm{meV}$. After the KamLAND-Zen 400, we have started KamLAND-Zen 800 project which will use $750 \mathrm{~kg}$ xenon and new clean nylon balloon called mini-balloon to contain xenon loaded liquid scintillator. We made mini-balloon with various contamination rejection techniques, and we successfully installed it in KamLAND. Currently we are purifying non-xenon-loaded scintillator in mini-balloon. After the purification, we will dissolve xenon in the liquid scintillator.
\end{abstract}

Neutrino Oscillation Workshop (NOW2018)

9 - 16 September, 2018

Rosa Marina (Ostuni, Brindisi, Italy)

\footnotetext{
* Speaker.

${ }^{\dagger}$ on behalf of the KamLAND-Zen collaboration.
} 


\section{Introduction}

Neutrinoless double beta decay $(0 v \beta \beta)$ is a key for the beyond the standard model of elementary particle physics. If we observe this decay, it means that neutrino is Majorana particle and it violates lepton number conservatoin. The half life of $0 v \beta \beta$ is proportional to the square of the effective neutrino mass $\left\langle m_{\beta \beta}\right\rangle$. Thus the event rate tells us mass scale of light neutrino mass. $0 \mathrm{v} \beta \beta$ emits two beta rays and the total energy is correspond to $Q$-value of double beta decay nuclei. Usually the Q-values are region in environmental backgrounds by Uranium-chain, Thorium-chain and Potassium. In order to observe the signal, we have to eliminate these noise events, or reduce radioactive source in/near detectors. Recently some experiments released the upper limits on $\left\langle m_{\beta \beta}\right\rangle$ of $\sim 60-300 \mathrm{meV}$, based on the each background rejection technique[1][2][3][4][5].

\section{KamLAND-Zen}

KamLAND-Zen is a double beta decay experiment with ${ }^{136} \mathrm{Xe}$ based on the anti-neutrino detector KamLAND[6]. Xenon loaded liquid scintillator (Xe-LS) is stored in nylon made miniballoon and it is kept in 1000 tons of liquid scintillator (LS) in 13m diameter outer balloon (Figure 1). Xe-LS can contain almost $3 \mathrm{wt} \%$ xenon gas which is enriched to $90.6 \%{ }^{136} \mathrm{Xe}$. The scintillation lights from $\beta \beta$ and other radiations ( $\alpha, \beta, \gamma, \mathrm{p}$, etc.) in Xe-LS are detected by 1879 photo multiplier tubes (PMTs). The vertex and energy are reconstructed by hit timing distribution of PMTs and transparency corrected number of photo-electrons. These resolutions are $14.1 \mathrm{~cm}$ and $6.6 \% \sim$ $7.3 \% \sqrt{\mathrm{E}(\mathrm{MeV})}$ at $\sigma$. Because of size of detector, KamLAND-Zen has scalability. Liquid scintillator type detector can achieve ultra-clean environment. KamLAND already has $10^{-17 \sim-18} \mathrm{~g} / \mathrm{g}$ contamination level for ${ }^{238} \mathrm{U}$ and ${ }^{232} \mathrm{Th}$ in liquid scintillator[7], then very clean container realize high sensitivity search for $0 v \beta \beta$. The container for Xe-LS called mini-balloon is made by $25 \mu \mathrm{m}$ thickness nylon film as shown in Figure 2. Nylon film has $99 \%$ transparency and contamination level is $\sim 2 \times 10^{-12} \mathrm{~g} / \mathrm{g}$ level for ${ }^{238} \mathrm{U}$ and ${ }^{232} \mathrm{Th}$. To make the drop shape container, nylon films were cut for each part and connected by heat welding in class 1 super clean room.

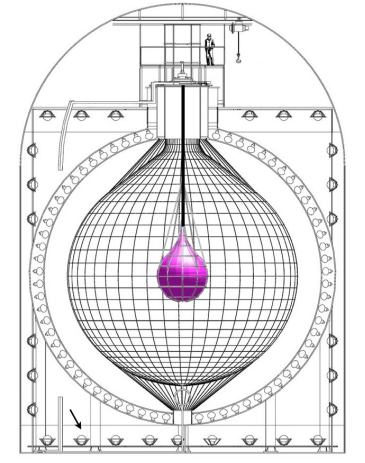

Figure 1: Schimatic view of KamLAND-Zen

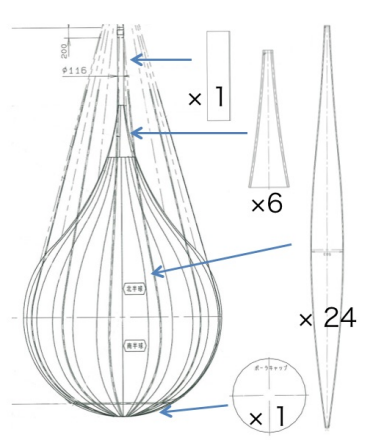

Figure 2: Each nylon film part for mini-balloon 


\section{KamLAND-Zen 400 results}

KamLAND-Zen 400 started data acquisition in October 2011 and terminated in October 2015, including purification period from June 2012 to December 2013. In the first phase before purification (Phase-I), we found ${ }^{110 m} \mathrm{Ag}$ events in the region of interest for $0 v \beta \beta$ (Figure 3)[8]. We suspected the impurities came from Fukushima reactor accident by earthquake and its tsunami. After the Phase-I, we purified xenon gas by distillation and getter filter after the extraction from Xe-LS. On the other hand, liquid scintillator after the rejection of xenon gas was purified three times by distillation, and replaced to new one twice. Unfortunately, inner surface of mini-balloon was contaminated from mine air by pump failure, thus effective fiducial volume was $\sim 40 \%$ at phase II. The energy spectrum of purified Xe-LS was shown in Figure 4 which has no peak of ${ }^{110 m} \mathrm{Ag}$. From the combined analysis with Phase-I and Phase-II, lower limit for half life of $0 v \beta \beta$ is $\mathrm{T}_{1 / 2}>1.07 \times 10^{26}$ years at $90 \%$ confidence level. It corresponds to $\left\langle m_{\beta \beta}\right\rangle<61-165 \mathrm{meV}$.

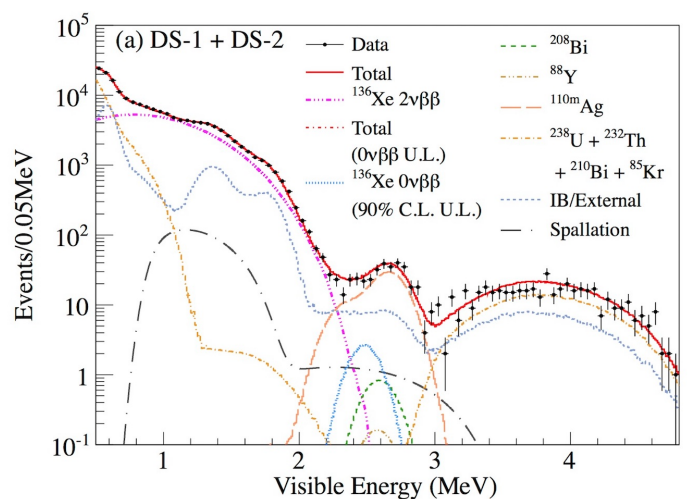

Figure 3: Energy spectrum in Phase-I

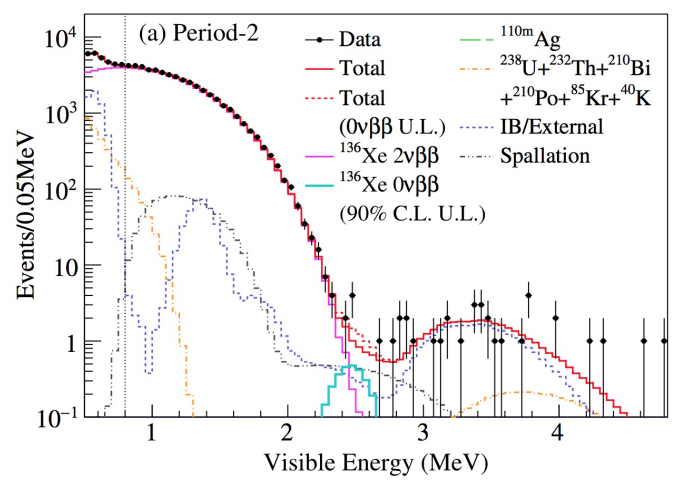

Figure 4: Energy spectrum in Phase-II

\section{KamLAND-Zen 800}

Because of the $\gamma$-rays from the surface contamination of mini-balloon, sensitivity was restricted in KamLAND-Zen 400. Therefore we started KamLAND-Zen 800 project with almost $750 \mathrm{~kg}$ xenon and cleaner mini-balloon. In order to make cleaner mini-balloon, we applied some techniques, clean wear control, particle flow check, static-electricity control by ion generation devices and humidity control, film cover setting for mini-balloon film, and semi-automatic welding machine introduction.

Mini-balloon production was done by following procedure. Nylon film washing by ultra pure water with ultra sonic cleaning to reject initial surface contaminations, film cover setting for miniballoon film, cutting for each part, film connection by welding, leak check by helium and helium detector, repairing for holes by glue. The production was started in May 2017, and preparation for delivery to experimental site kamioka was done at April 2018.

Installation of mini-balloon to KamLAND was done at May 10, 2018 (Figure 5). For the install preparations in Kamioka site, we set class 50 level clean room on the KamLAND. KamLAND is spherical detector and access point to inside of outer balloon is only $50 \mathrm{~cm}$ diameter at top area. 
Thus we folded mini-balloon and kept the shape by perforated teflon sheet and teflon tubes. We also applied cover nylon films between mini-balloon film and teflon sheets to avoid damages at the installation works. We installed mini-balloon with heavier (+0.4\%) LS compared to the KamLAND LS density. After the sink of mini-balloon in KamLAND LS, teflon sheets and cover nylon films were removed and pulled up. After the installation, we filled slightly heavier LS $(+0.015 \%)$ without xenon, and mini-balloon was expanded as shown in Figure 6.

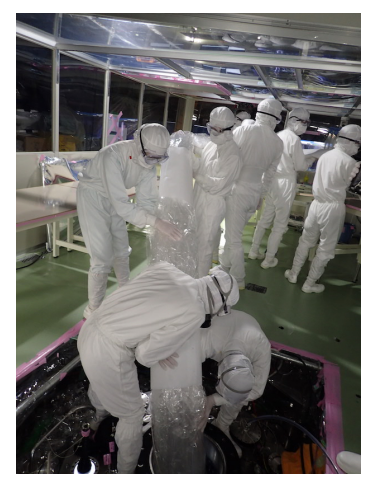

Figure 5: mini-balloon installation

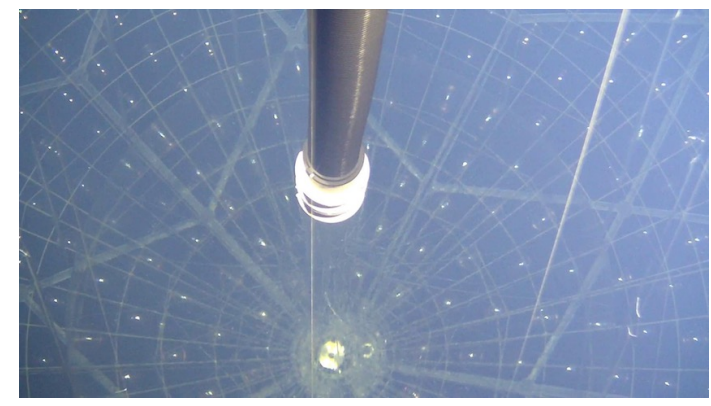

Figure 6: Expanded mini-balloon in KamLAND

We have checked quality of non xenon loaded LS, and we estimated ${ }^{232} \mathrm{Th}$ level is $\mathrm{O}\left(10^{-15}\right)$ $\mathrm{g} / \mathrm{g}$. This contamination level is not so affect to the sensitivity for $0 v \beta \beta$ search. But overlaid events in same event time window by ${ }^{212} \mathrm{Bi}$ and ${ }^{212} \mathrm{Po}(\tau=430 \mathrm{~ns})$ in ${ }^{232} \mathrm{Th}$-chain will be in ROI. Therefore we are purifying the LS to achieve best performance. After the purification, we will dissolve xenon gas to the LS and we will start physics run in this winter.

\section{Conclusions}

The lower limit for half life of $0 v \beta \beta$ of ${ }^{136} \mathrm{Xe}$ is $\mathrm{T}_{1 / 2}>1.07 \times 10^{26}$ years at $90 \%$ confidence level by KamLAND-Zen 400 result. It corresponds to $\left\langle m_{\beta \beta}\right\rangle<61-165 \mathrm{meV}$. We are preparing to start KamLAND-Zen 800 physics run. Mini-balloon was produced using various low-background techniques, and mini-balloon was successfully installed. KamLAND-Zen 800 data acquisition will commence in this winter.

\section{References}

[1] A.Gando et al. (KamLAND-Zen Collaboration), Phys. Rev. Lett. 117, 082503 (2016).

[2] J. B. Albert et al. (EXO Collaboration) Phys. Rev. Lett. 120072701 (2018).

[3] C. E. Aalseth et al. (MAJORANA Collaboration) Phys. Rev. Lett. 120132502 (2018).

[4] M. Agostini et al. (GERDA Collaboration) Phys. Rev. Lett. 120132503 (2018).

[5] C. Alduino et al. (CUORE Collaboration) Phys. Rev. Lett. 120, 132501 (2018).

[6] K. Eguchi et al. (KamLAND Collaboration) Phys. Rev. Lett. 90021802 (2003).

[7] A.Gando et al. (KamLAND Collaboration) Phys. Rev. C 92, 055808 (2015).

[8] A.Gando et al. (KamLAND-Zen Collaboration), Phys. Rev. C 85, 045504 (2012). 